\title{
INVERSE HEAT TRANSPORT PROBLEMS FOR COEFFICIENTS IN TWO-LAYER DOMAINS AND METHODS FOR THEIR SOLUTION
}

\author{
S. GUSEINOV and A. BUIKIS \\ Institute of Mathematics, Latvian Academy of Sciences and University of \\ Latvia
}

1 Akademijas Square, Riga, LV-1524, LATVIA

E-mail: sharif@one.lv, buikis@latnet.lv

Received October 1, 2002

\begin{abstract}
In various fields of science and technology it is often necessary to solve inverse problems, where from measurements of state of the system or process it is required to determine a certain typesetting of the causal characteristics. It is known that infringement of the natural causal relationships can entail incorrectness of the mathematical stating of inverse problems. Therefore the development of efficient methods for solving such problems allows one to considerably simplify experimental research and to increase the accuracy and reliability of the obtained results due to certain complication of algorithms for processing the experimental data. The problem of determination of thermal diffusivity coefficients considering other known characteristics of heat transport process is among incorrect inverse problems. These inverse problems for coefficients are quite difficult even in the case of homogeneous media. In this paper it is supposed that the heat transport equation is non-homogeneous and an algorithm for determination of the thermal diffusivity coefficients for both the media is proposed. At the first step, the non-homogeneous inverse problem with piecewise-constant function of non-homogeneity is solved. For this auxiliary inverse problem, the proposed method allows one to determine both the coefficients of thermal diffusivity and to restore the heat transport process without any additional information, i.e. the algorithm also solves the direct problem. Then the initial non-homogeneous inverse problem with a piecewise-continuous function of non-homogeneity is solved. The proposed method reduces the non-homogeneous inverse problem for coefficients to a set of two transcendent algebraic equations. Finally, the analytical solution to direct problem is obtained using Green's function.
\end{abstract}

Key words: inverse problems, heat transport, multi-layered domains. 


\section{INTRODUCTION}

The problem of determination of the thermal diffusivity coefficient considering other characteristics of heat transport process is among the incorrect inverse problems. This inverse problem for coefficients are quite difficult even in case of homogeneous medium. The "Transient Hot - Strip Method" (THS Method), offered in the paper [2], is a well-known one for determination of the coefficient of thermal diffusivity and the specific heat in homogeneous media, assuming other conditions of heat transport process are given. In works $[3 ; 5]$ numerical variants of THS Method for various boundary conditions are investigated. In work [4] a numerical method (finite element method) for the analysis of two - layer medium is generalized. In our paper [1], distinguished from the mentioned works $[2 ; 3 ; 5]$, it was assumed that the medium is two layered in the direction perpendicular to the "Hot - Strip" surface and a qualitative new mathematical method is offered. It allows one to determine both the coefficients of thermal diffusivity for measurements of the temperature in some suitable from the experiment point of view points. In the first part of this paper we briefly explain the results of our work [1], in the second part of the paper the new technique is developed. The proposed method reduces the non-homogeneous inverse problem for coefficients to a set of two transcendent algebraic equations without additional experimental information.

\section{THE STATEMENT OF THE HOMOGENEOUS INVERSE PROBLEM}

Geometrically this two - layer domain looks as follows

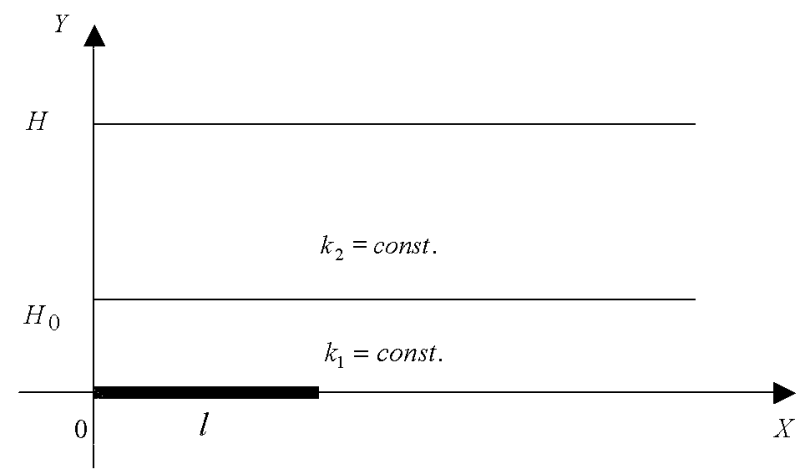

Figure 1. 
The problem is formulated mathematically as follows:

$$
\begin{aligned}
& c(y) u_{t}(x, y, t)=k^{2}(y)\left(u_{x x}(x, y, t)+u_{y y}(x, y, t)\right), \\
& \left.u\right|_{y=0}=h=\text { const }, \\
& \left.u_{y}\right|_{y=0}= \begin{cases}q, & 0<x \leq l, \\
0, & l<x<\infty,\end{cases} \\
& \left.u_{y}\right|_{y=H}=0, \\
& \left.u_{x}\right|_{x=0}=0 .
\end{aligned}
$$

To the above conditions there are added those of conjugation along the boundary $y=H_{0}$ of both media:

$$
\begin{aligned}
& \left.u\right|_{y=H_{0}-0}=\left.u\right|_{y=H_{0}+0}, \\
& \left.k_{1} u_{y}\right|_{y=H_{0}-0}=\left.k_{2} u_{y}\right|_{y=H_{0}+0} .
\end{aligned}
$$

Here the specific heat $c(y)$ per volume unit is given by

$$
c(y)= \begin{cases}c_{1}, & 0<y<H_{0} \\ c_{2}, & H_{0}<y<H\end{cases}
$$

and heat conductivity coefficient

$$
k(y)= \begin{cases}k_{1}, & 0<y<H_{0} \\ k_{2}, & H_{0}<y<H\end{cases}
$$

The purpose of the paper is to define the coefficient $a^{2}(y)=\frac{k(y)}{c(y)}$, that is to find two numerical values $-a_{1}^{2}$ and $a_{2}^{2}$.

\section{THE SOLUTION OF HOMOGENEOUS INVERSE PROBLEM USING INTEGRAL LAPLACE'S TRANSFORMATION}

We begin to solve the stated problem rewriting the boundary condition (2.5) in the following equivalent form:

$$
\left.\left[(x-\xi) u_{\xi}(\xi, \eta, t)\right]\right|_{\xi=0} ^{\xi=x}=0
$$


which, in the end, can be written as

$$
\int_{0}^{x}(x-\xi) u_{\xi \xi} d \xi=u(x, \eta, t)-u(0, \eta, t)
$$

In a similar way the boundary condition (2.3) can be transformed:

$$
\int_{0}^{y}(y-\eta) u_{\eta \eta} d \eta=u(\xi, y, t)-u(\xi, 0, t)+ \begin{cases}-q y, & 0<x \leq l \\ 0, & x>l\end{cases}
$$

Now we multiply $(3.1)$ by $(y-\eta)$ and integrate it over $\eta$ and similarly, multiplying (3.2) by $(x-\xi)$ and integrating it over $\xi$ and summing up both equalities, we obtain

$$
\begin{aligned}
\int_{0}^{x}(x-\xi)\left[\int_{0}^{y}(y-\eta) \frac{u_{t}(\xi, \eta, t)}{a^{2}(\eta)} d \eta-u(\xi, y, t)+u(\xi, 0, t)\right] d \xi= \\
\quad=\int_{0}^{y}(y-\eta)[u(x, \eta, t)-u(0, \eta, t)] d \eta+ \begin{cases}-\frac{1}{2} x^{2} y q, & 0<x<l \\
0, & x>l .\end{cases}
\end{aligned}
$$

Assuming time $t$ to be fixed, we rewrite the last equality in the following short form:

$$
\int_{0}^{x}(x-\xi) f_{1}(\xi, y, t) \mathrm{d} \xi=f_{2}(x, y, t)+ \begin{cases}-\frac{1}{2} x^{2} y q, & 0<x \leq l \\ 0, & x>l\end{cases}
$$

here

$$
\left\{\begin{array}{l}
f_{1}(\xi, y, t)=\int_{0}^{y}(y-\eta) \frac{u_{t}(\xi, \eta, t)}{a^{2}(\eta)} d \eta-u(\xi, y, t)+u(\xi, 0, t) \\
f_{2}(x, y, t)=\int_{0}^{y}(y-\eta)[u(x, \eta, t)-u(0, \eta, t)] d \eta
\end{array}\right.
$$

Expression (3.3) is an integro-differential Volterra type equation, since $f_{1}(\xi, y, t)$ contains both the function $u(\xi, \eta, t)$ and its derivative with respect to $t$, while function $f_{2}(x, y, t)$ contains $u(x, \eta, t)$ under the integration sign. We will solve equation (3.3) using integral Laplace's transform:

$$
\tilde{g}(p)=\int_{0}^{\infty} g(x) e^{-p x} d x, \quad g(x)=\frac{1}{2 \pi i} \int_{\sigma-i \infty}^{\sigma+i \infty} \tilde{g}(p) e^{p x} d p
$$


After simple transformations we finally receive

$$
\begin{gathered}
\int_{0}^{y}(y-\eta) \frac{\tilde{u}_{t}(p, \eta, t)}{a^{2}(\eta)} d \eta-\tilde{u}(p, y, t)+\tilde{u}(p, 0, t)=p^{2} \int_{0}^{y}(y-\eta) \tilde{u}(p, \eta, t) d \eta \\
-p \int_{0}^{y}(y-\eta) u(0, \eta, t) d \eta+y q \frac{e^{-p l}}{p}\left(\frac{l^{2}}{2} p^{2}+l p+1-e^{-p l}\right) .
\end{gathered}
$$

The obtained equation presents an integro-differential Volterra II kind equation. It is well known that the solution of (3.4) exists and it is unique. This solution is expressed via a resolvent, with not only $a^{2}(y)$ being found but also $u(x, y, t)$.

\section{THE SOLUTION OF HOMOGENEOUS INVERSE PROB- LEM HAVING INFORMATION ABOUT TEMPERATURE AT SOME POINTS}

In this Section we will narrow down our task aiming only at finding $a_{1}^{2}$ and $a_{2}^{2}$. With this purpose in mind, we return to (3.1) and (3.2), substituting $x=l$, and $\eta=\eta_{0} \in\left[0, H_{0}\right]$ into $(3.1)$ and $\xi=l$ and $y=y_{0} \in\left[H_{0}, H\right]$ into (3.2). We thus obtain:

$$
\begin{aligned}
& \int_{0}^{l}(l-\xi) u_{\xi \xi}\left(\xi, \eta_{0}, \tau\right) d \xi=u\left(l, \eta_{0}, \tau\right)-u\left(0, \eta_{0}, \tau\right), \\
& \int_{0}^{l}\left(y_{0}-\eta\right) u_{\eta \eta}(l, \eta, \tau) d \eta=u\left(l, y_{0}, \tau\right)-u(l, 0, \tau)-y_{0} q .
\end{aligned}
$$

Note that problem (4.1), (4.2) is ill posed: small variations in the right sides correspond to arbitrarily large variations in the solution. For solving such problems, a special regularization method was developed. Let us suppose that, using the regularization method, we have found solutions of (4.1) as

$$
u_{\xi \xi}\left(\xi, \eta_{0}, \tau\right)=z_{1}^{(0)}\left(\xi, l, u\left(l, \eta_{0}, \tau\right), u\left(0, \eta_{0}, \tau\right)\right)
$$

and of (4.2) as

$$
u_{\eta \eta}(l, \eta, \tau)=z_{2}^{(0)}\left(\eta, y_{0}, u\left(l, y_{0}, \tau\right), u(l, 0, \tau)\right)
$$

Then, integrating the initial equation (2.1) over time and setting $\xi=l$ we obtain

$$
u\left(l, \eta_{0}, T\right)-h=a_{1}^{2} \int_{0}^{T}\left[u_{\xi \xi}\left(l, \eta_{0}, \tau\right)+u_{\eta \eta}\left(l, \eta_{0}, \tau\right)\right] d \tau
$$


From here it follows, by virtue of (4.3) and (4.4), that

$$
a_{1}^{2}=\frac{u\left(l, \eta_{0}, T\right)-h}{\int_{0}^{T}\left[z_{1}^{(0)}\left(l, l, u\left(l, \eta_{0}, \tau\right), u\left(0, \eta_{0}, \tau\right)\right)+z_{2}^{(0)}\left(\eta_{0}, y_{0}, u\left(l, y_{0}, \tau\right), u(l, 0, \tau)\right)\right] \mathrm{d} \tau} .
$$

This means that, with temperature values at the points $\left(x=l, y=\eta_{0}\right)$ and $\left(x=0, y=\eta_{0}\right)$ being known for some time interval $\tau \in[0, T]$, from (4.3) we can reconstruct the function $u_{\xi \xi}\left(\xi, \eta_{0}, \tau\right)$. Correspondingly, from given temperature at the points $\left(x=l, y=y_{0}\right)$ and $(x=l, y=0)$ function $u_{\eta \eta}(l, \eta, \tau)$ can be reconstructed. To find $a_{2}^{2}$, we will set: $\xi=l$ and $\eta=\eta_{1} \in\left[H_{0}, H\right]$. Then, instead of (4.5) we will have

$$
u\left(l, \eta_{1}, T\right)-h=a_{2}^{2} \int_{0}^{T}\left[u_{\xi \xi}\left(l, \eta_{1}, \tau\right)+u_{\eta \eta}\left(l, \eta_{1}, \tau\right)\right] d \tau
$$

and instead of (4.1)

$$
\int_{0}^{l}(l-\xi) u_{\xi \xi}\left(\xi, \eta_{1}, \tau\right) d \xi=u\left(l, \eta_{1}, \tau\right)-u\left(0, \eta_{1}, \tau\right) .
$$

We denote the solution of this last equation as $u_{\xi \xi}\left(\xi, \eta_{1}, \tau\right)=$ $z_{1}^{(1)}\left(\xi, l, u\left(l, \eta_{1}, \tau\right), u\left(0, \eta_{1}, \tau\right)\right)$. Next, if the solution of (4.4) at the point $\eta=\eta_{1}$ is denoted by $z_{2}^{(1)}$, that is $u_{\eta \eta}\left(l, \eta_{1}, \tau\right)=z_{2}^{(1)}\left(\eta_{1}, y_{0}, u\left(l, y_{0}, \tau\right), u(l, 0, \tau)\right)$, then (4.6) can be rewritten as

$$
a_{2}^{2}=\frac{u\left(l, \eta_{1}, T\right)-h}{\int_{0}^{T}\left[z_{1}^{(1)}\left(l, l, u\left(l, \eta_{1}, \tau\right), u\left(0, \eta_{1}, \tau\right)\right)+z_{2}^{(1)}\left(\eta_{1}, y_{0}, u\left(l, y_{0}, \tau\right), u(l, 0, \tau)\right)\right] d \tau} .
$$

Then it follows that solutions of integral equations $z_{i}^{(j)}, i=1,2 ; j=0,1$, depend on the temperatures measured in $\operatorname{six}(x, y)$ points only: $\left\{0, \eta_{0}\right\},\left\{0, \eta_{1}\right\}$, $\{l, 0\},\left\{l, \eta_{0}\right\},\left\{l, \eta_{1}\right\},\left\{l, y_{0}\right\}$; we are reminded, though, that the parameters $\eta_{0} \in\left[0, H_{0},\right], \eta_{1} \in\left[H_{0}, H\right]$ and $y_{0} \in\left[H_{0}, H\right]$ from the corresponding segments might be chosen arbitrarily in the manner that is convenient from the experimental point of view. In particular, the number of such points can be reduced to four if we set $\eta_{0}=0, \eta_{1}=y_{0}=H$.

\section{THE SOLUTION OF INHOMOGENEOUS INVERSE PROB- LEM USING GREEN'S FUNCTION}

In this paper, distinguished from our paper [1], it is supposed that considered problem is non-uniform. Besides, in this problem there are no data on tem- 
Inverse heat transport problems for coefficients in two-layer domains 223

perature in points of the considered area. So, we have the following problem:

$$
\begin{aligned}
& c(y) u_{t}(x, y, t)=k(y)\left[u_{x x}(x, y, t)+y_{y y}(x, y, t)\right]+\tilde{f}(x, y) \\
& 0<x<+\infty, 0<y<H=H_{0}+H_{1}, t>0 \text {, } \\
& \tilde{f}(x, y)=\left\{\begin{array}{lll}
f(x), & \text { if } \quad 0<y<H_{0}, \\
0, & \text { if } \quad H_{0}<y<H,
\end{array}\right. \\
& \left.u\right|_{t=0}=h=\text { const }, \quad 0<x<\infty, 0<y<H, \\
& \left.u_{y}\right|_{y=0}=0, \quad 0<x<\infty, t>0 \text {, } \\
& \left.u_{y}\right|_{y=H}=0, \quad 0<x<\infty, t>0 \text {, } \\
& \left.u_{x}\right|_{x=0}=0, \quad 0<y<H, t>0, \\
& \left\{\begin{array}{l}
\left.u\right|_{y=H_{0}-0}=\left.u\right|_{y=H_{0}+0}, \\
\left.k_{1} u_{y}\right|_{y=H_{0}-0}=\left.k_{2} u_{y}\right|_{y=H_{0}+0}
\end{array}\right.
\end{aligned}
$$

In addition it is supposed, that the following function is known $\frac{1}{l} \int_{0}^{l} u(x, 0, t) d x=$ $T(t)$. Let's denote $\theta(x, y, t)=u(x, y, t)-h$. Then we receive the following equivalent problem:

$$
\theta_{t}(x, y, t)=a^{2}(y)\left[\theta_{x x}(x, y, t)+\theta_{y y}(x, y, t)\right]+f(x, y),
$$

where

$$
\begin{aligned}
& f(x, y)=\frac{\tilde{f}(x, y)}{c(y)} \\
& \left.\theta\right|_{t=0}=0, \quad 0<x<+\infty, \quad 0<y<H, \\
& \left.\theta_{y}\right|_{y=0}=0, \quad 0<x<+\infty, \quad t>0, \\
& \left.\theta_{y}\right|_{y=H}=0, \quad 0<x<+\infty, \quad t>0, \\
& \left.\theta_{x}\right|_{x=0}=0, \quad 0<y<H, \quad t>0, \\
& \left.\theta\right|_{y=H_{0}-0}=\left.\theta_{y}\right|_{y=H_{0}+0} \\
& \left.k_{1} \theta_{y}\right|_{y=H_{0}-0}=\left.k_{2} \theta_{y}\right|_{y=H_{0}+0}, \\
& T_{1}(t)=\frac{1}{l} \int_{0}^{l} \theta(x, 0, t) d x=T(t)-h .
\end{aligned}
$$


First we assume to have the homogeneous equation and we formally apply to (5.4) - (5.11) Fourier cosine-transform on $x$ :

$$
\begin{aligned}
& \tilde{\theta}_{t}=a^{2}(y)\left[\tilde{\theta}_{y y}-\lambda^{2} \tilde{\theta}\right] \\
& \tilde{\theta}(\lambda, y, t)=e^{-a^{2}(y) \lambda^{2} t} V(\lambda, y, t), \\
& V_{t}=a^{2}(y) V_{y y}, \quad 0<y<H, \quad t>0 \\
& \left.V\right|_{t=0}=0, \\
& \left.V_{y}\right|_{y=0}=0,\left.\quad V_{y}\right|_{y=H}=0, \\
& V\left(\lambda, H_{0}-0, t\right)=V\left(\lambda, H_{0}+0, t\right) e^{\left(a_{1}^{2}-a_{2}^{2}\right) \lambda^{2} t}, \\
& k_{1} V_{y}\left(\lambda, H_{0}-0, t\right)=k_{2} V_{y}\left(\lambda, H_{0}+0, t\right) e^{\left(a_{1}^{2}-a_{2}^{2}\right) \lambda^{2} t} .
\end{aligned}
$$

This problem is solved by the method of separation of variables:

$$
Y_{n}(y)=\left\{\begin{array}{l}
\frac{}{\frac{\cos \frac{\omega_{n}}{a_{1}} y}{\cos \frac{\omega_{n}}{a_{1}} H_{0}}, \quad \text { if } \quad 0<y<H_{0},} \\
\frac{\cos \frac{\omega_{n}}{a_{2}}(H-y)}{\cos \frac{\omega_{n}}{a_{1}}\left(H-H_{0}\right)}, \quad \text { if } \quad H_{0}<y<H \\
\tilde{T}^{(n)}(t)=D_{n} e^{-\left(\omega_{n}^{2}+a^{2}(y) \lambda^{2}\right) t},
\end{array}\right.
$$

where $\omega=\omega_{n}$ are solutions of the transcendental equation

$$
\begin{aligned}
& c_{1} a_{1} \tan \frac{\omega}{a_{1}} H_{0}=c_{2} a_{2} \tan \frac{\omega}{a_{2}}\left(H_{0}-H\right), \\
& D_{n}=\frac{\int_{0}^{H} c(\eta) \tilde{h}(\lambda, \eta) Y_{n}(\eta) d \eta}{\left\|Y_{n}\right\|^{2}}, \quad\left\|Y_{n}\right\|^{2}=\int_{0}^{H} c(\eta) Y_{n}^{2}(\eta) d \eta .
\end{aligned}
$$

After application of Fourier inverse cosine-transform we receive

$$
\begin{aligned}
\theta(x, y, t)=\frac{h}{2 a \sqrt{\pi t}} \int_{0}^{\infty} d \xi \int_{0}^{H} c(\eta)\left[\frac{1}{\left\|Y_{0}\right\|^{2}}\right. & \left.+\sum_{n=1}^{\infty} \frac{e^{-\omega_{n}^{2} t}}{\left\|Y_{n}\right\|^{2}} Y_{n}(y) Y_{n}(\eta)\right] \\
& \times\left[e^{-\frac{(x-\xi)^{2}}{4 a^{2} t}}+e^{-\frac{(x+\xi)^{2}}{4 a^{2} t}}\right] d \eta .
\end{aligned}
$$


We denote through $G(a(y), c(\eta) ; x, y, t ; \xi, \eta, \tau)$ Green's function of the problem (2.1), (5.4) - (5.11), where $0<\tau<t$. Then

$$
u(x, y, t)=\int_{0}^{t} \int_{0}^{\infty} \int_{0}^{H} G(a(y), c(\eta) ; x, y, t ; \xi, \eta, \tau) f(\xi, \eta) d \tau d \xi d \eta,
$$

where

$$
\begin{aligned}
G(a(y), c(\eta) ; x, y, t ; \xi, \eta, \tau)=c(\eta) & \frac{e^{-\frac{(x-\xi)^{2}}{4 a^{2}(t-\tau)}}+e^{-\frac{(x+\xi)^{2}}{4 a^{2}(t-\tau)}}}{2 a(y) \sqrt{\pi(t-\tau)}} \\
& \times\left[\frac{1}{\left\|Y_{0}\right\|^{2}}+\sum_{n=1}^{\infty} \frac{e^{-\omega_{n}^{2}(t-\tau)}}{\left\|Y_{n}\right\|^{2}} Y_{n}(y) Y_{n}(\eta)\right]
\end{aligned}
$$

and

$$
\begin{aligned}
& \left\|Y_{0}\right\|^{2}=c_{1} H_{0}+c_{2}\left(H-H_{0}\right), \\
& \left\|Y_{n}\right\|^{2}=\int_{0}^{H} c(\eta) Y_{n}^{2}(\eta) d \eta=\frac{c_{1} H_{0}}{2 \cos ^{2} \frac{\omega_{n}}{a_{1}} H_{0}}+\frac{c_{2}\left(H-H_{0}\right)}{2 \cos ^{2} \frac{\omega_{n}}{a_{2}}\left(H-H_{0}\right)} .
\end{aligned}
$$

From (5.5) it follows that

$$
\begin{gathered}
\theta(x, y, t)=h+\frac{c_{1} H_{0}}{2 \sqrt{\pi} a(y)\left\|Y_{0}\right\|^{2}} \int_{0}^{t} \int_{0}^{\infty} \frac{e^{-\frac{(x-\xi)^{2}}{4 a^{2}(y)(t-\tau)}}+e^{-\frac{(x+\xi)^{2}}{4 a^{2}(y)(t-\tau)}}}{\sqrt{t-\tau}} f(\xi) d \xi d \tau \\
+\frac{c_{1} a_{1}}{2 \sqrt{\pi} a(y)\left\|Y_{0}\right\|^{2}} \int_{0}^{t} \int_{0}^{\infty} \frac{e^{-\frac{(x-\xi)^{2}}{4 a^{2}(y)(t-\tau)}}+e^{-\frac{(x+\xi)^{2}}{4 a^{2}(y)(t-\tau)}} \tilde{g}(y, t-\tau) f(\xi) \mathrm{d} \xi \mathrm{d} \tau .}{\sqrt{t-\tau}}
\end{gathered}
$$

Here

$$
\tilde{g}(y, t-\tau)=\sum_{n=1}^{\infty} \frac{\tan \frac{\omega_{n}}{a_{1}} H_{0}}{\omega_{n}\left\|Y_{n}\right\|^{2}} Y_{n}(y) e^{-\omega_{n}^{2}(t-\tau)}
$$

and it depends from $a_{1}$ and $a_{2}$. In the beginning we assume, that the function $f(x, y)$ in $(5.5)$ is constant, i.e.

$$
f(x, y)=\left\{\begin{array}{lll}
f(x)=f_{0}, & \text { if } \quad 0<y<H_{0} \\
0, & \text { if } \quad H_{0}<y<H
\end{array}\right.
$$


Then we have

$$
\theta(x, y, t)=\frac{c_{1} H_{0} f_{0}}{\left\|Y_{0}\right\|^{2}} t+c_{1} a_{1} f_{0} \int_{0}^{t} \tilde{g}(y, \tau) d \tau
$$

Fixing the moment of time, we receive

$$
\frac{c_{1} H_{0} f_{0}}{\left\|Y_{0}\right\|^{2}} t_{0}+c_{1} a_{1} f_{0} g\left(a_{1}, a_{2}, t_{0}\right)=T\left(t_{0}\right)-h
$$

where

$$
g\left(a_{1}, a_{2}, t_{0}\right)=\sum_{n=1}^{\infty} \frac{\tan \frac{\omega_{n}}{a_{1}} H_{0}}{\omega_{n}^{3}\left\|Y_{n}\right\|^{2} \cos \frac{\omega_{n}}{a_{1}} H_{0}}\left[1-e^{-\omega_{n}^{2} t_{0}}\right]
$$

or in another way

$$
g\left(a_{1}, a_{2}, t_{0}\right)=\int_{0}^{t_{0}} \tilde{g}(0, \tau) d \tau
$$

The equality (5.15) is the first transcendental equation for definition $a_{1}$ and $a_{2}$. The second transcendental equation for definition also can be received from the second condition of conjugation (2.7):

$$
\begin{aligned}
c_{1} a_{1} H_{0} \sum_{n=1}^{\infty} \frac{\tan ^{2} \frac{\omega_{n}}{a_{1}} H_{0}}{\omega_{n}^{2}\left\|Y_{n}\right\|^{2}}\left[1-e^{-\omega_{n}^{2} t}\right]=-c_{2} a_{2}\left(H-H_{0}\right) \\
\times \sum_{n=1}^{\infty} \frac{\tan \frac{\omega_{n}}{a_{1}} H_{0} \tan \frac{\omega_{n}}{a_{2}}\left(H-H_{0}\right)}{\omega_{n}^{2}\left\|Y_{n}\right\|^{2}}\left[1-e^{-\omega_{n}^{2} t}\right] .
\end{aligned}
$$

So, coefficients $a_{1}$ and $a_{2}$ can be found from the transcendental equations (5.15) and (5.16). Initial function of heat conductivity is defined in the field of $\{0<x<\infty, 0<y<H, t>0\}$ by following formula:

$$
u(x, y, t)=h+\frac{c_{1} H_{0} f_{0}}{\left\|Y_{0}\right\|^{2}} t+c_{1} a_{1} f_{0} \int_{0}^{t} \tilde{g}(y, \tau) d \tau .
$$

In a case when function $f(x, y)$ is not constant we shall receive, similarly

$$
\int_{0}^{t_{0}} \int_{0}^{\infty}[1+g(\tau)]\left[\operatorname{erf}\left(\frac{\xi+l}{2 a_{1} \sqrt{\tau}}\right)+\operatorname{erf}\left(\frac{l-\xi}{2 a_{1} \sqrt{\tau}}\right)\right] f(\xi) d \xi d \tau=\frac{2 l\left\|Y_{0}\right\|^{2}}{c_{1} H_{0}}\left[T\left(t_{0}\right)-h\right]
$$


where

$$
g(\tau)=\frac{a_{1}\left\|Y_{0}\right\|^{2}}{H_{0}} \sum_{n=1}^{\infty} \frac{\tan \frac{\omega_{n}}{a_{1}} H_{0}}{\omega_{n}\left\|Y_{n}\right\|^{2} \cos \frac{\omega_{n}}{a_{1}} H_{0}} e^{-\omega_{n}^{2} \tau}
$$

and $\operatorname{erf}(z)=\frac{2}{\sqrt{\pi}} \int_{0}^{z} e^{-a^{2}} d a$ is the error integral.

Next we transform (5.17) by using the following formula

$$
\begin{aligned}
\int \frac{1}{x^{2 n+1}} \operatorname{erf}(a x) d x & =-\frac{1}{2 n x^{2 n}} \operatorname{erf}(a x)+\frac{1}{2 a n \sqrt{\pi \Gamma\left(n+\frac{1}{2}\right)}} \sum_{k=1}^{n}(-1)^{k} e^{-a^{2} x^{2}} \\
& \times \Gamma\left(n-k+\frac{1}{2}\right) a^{2 k} x^{2 k-2 n-1}+\frac{(-1)^{n} a^{2 n} \sqrt{\pi}}{2 n \Gamma\left(n+\frac{1}{2}\right)} \operatorname{erf}(a x)
\end{aligned}
$$

where $\Gamma(z)=\int_{0}^{+\infty} \lambda^{z-1} e^{-\lambda} d \lambda$ is Gamma function. Then we receive

$$
\int_{0}^{t_{0}} \int_{0}^{\infty}\left[\operatorname{erf}\left(\frac{\xi+l}{2 a_{1} \sqrt{\tau}}+\operatorname{erf}\left(\frac{l-\xi}{2 a_{1} \sqrt{\tau}}\right)\right] g(\tau) f(\xi) \mathrm{d} \xi \mathrm{d} \tau=\frac{2 l\left\|Y_{0}\right\|^{2}}{c_{1} H_{0}}\left[h-T\left(t_{0}\right)\right]\right.
$$

In this equality left and right parts are known if functions $f(x)$ and $T(x)$ are known. And also, the left part contains unknown parameters $a_{1}$ and $a_{2}$. So, for the concrete given $f(x)$, this equation is also the transcendental equation for definition $a_{1}$ and $a_{2}$. The second transcendental equation was found in the form of (5.16).

\section{CONCLUSION}

Thereby we have reduced the non-homogeneous inverse problem for coefficients to a set of two transcendent algebraic equations. Finally, the analytical solution of the direct problem can be obtained using Green's function.

\section{REFERENCES}

[1] Sh. Guseinov and A. Buikis. Calculation of the thermal diffusivity for two layer media. Latvian Journal of Physics and Technical Science, 4, 17 - 26, 2001.

[2] S. Gustafsson, E. Karawacki and M. Khan. Transient Hot - Strip Method for simultaneously measuring thermal conductivity and thermal diffusivity of solids and fluids. Journal Phys. D.: Appl. Phys., 12, 1411 - 1421, 1979.

[3] U. Hammerschmidt and W. Sabuga. Transient Hot - Strip (THS) Method: Uncertainty Assessment. International Journal of Thermophysic, 21(1), $217-247,2000$.

[4] R. Model and U. Hammerschmidt. An identification procedure for thermal transport properties of layered solids by means of transient measurements, 11p. (unpublished) 
[5] R. Model and U. Hammerschmidt. Numerical methods for the determination of thermal properties by means of transient measurements. Advanced Computational Methods in Heat Transfer, 6, $407-416,2000$.

\section{Atvirkštiniai šilumos laidumo uždaviniai dvisluoksnèse srityse ir ju sprendimo metodai}

S. Guseinov, A. Buikis

Ivairiose mokslo ir technologijos srityse dażnai tenka spręsti atvirkštinius użdavinius, kada remiantis sistemos ar proceso būsenos parametrų matavimais reikia nustatyti priežastines charakteristikas. Yra żinoma, kad natūralių prieżastinių priklausomybių nepaisymas gali nulemti neteisingą atvirkštinio użdavinio matematinę formuluotę. Todèl efektyvūs tokių użdavinių sprendimo metodai leis żymiai supaprastinti eksperimentinius tyrimus, padidinti gaunamų rezultatų tikslumą ir patikimumą, jeigu bus pritaikyti tam tikri sudètingesni eksperimentinių rezultatų apdorojimo būdai. Difuzijos koeficientu nustatymas naudojant kitas żinomas šilumos laidumo proceso charakteristikas priklauso nekorektiškų użdavinių kategorijai. Atvirkštiniai użdaviniai koeficientams yra sudètingi net ir homogeninèse terpèse. Šiame darbe daroma prielaida, kad terpè nehomogeniška, ir pasiūlytas algoritmas difuzijos koeficientų nustatymui tokiu atveju. Pirmajame etape sumażinamas nehomogeninis atvirkštinis użdavinys, laikant, kad nehomogeniškumas aprašomas dalimis pastoviomis funkcijomis. Šiam pagalbiniam atvirkštiniam użdaviniui siūlomas metodas leidžia apibrèżti abu šilumos difuzijos koeficientus ir atkurti šilumos laidumo proceso eigą be papildomos informacijos, t.y., algoritmas sprendżia taip pat ir tiesioginị użdavinį. Po to yra sprendżiamas atvirkštinis pradinis użdavinys esant dalimis tolydżiai nehomogeniškumą aprašančiai funkcijai. Siūlomas metodas redukuoja nehomogenini atvirkštini użdavinį i dviejų transcendentinių lygčių sprendimą. Taip pat yra gautas tiesioginio użdavinio analizinis sprendinys, taikant Gryno formulę. 